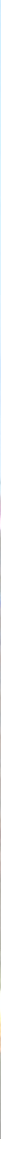

Figura 1. Edificios de construcción reciente en comunas de la ciudad de Santiago de Chile.

\title{
La transformación distinta a la forma. Contingencias no arquitectónicas sobre edificios en altura en la prensa chilena (2009-2017)
}

A transformação além da forma. Contingências não arquitetônicas sobre edifícios em altura na imprensa chilena (2009-2017)

The transformation beyond the form. Non-architectural contingencies on high-rise buildings in the Chilean press (2009-2017)

\section{Jorge Eduardo Vergara-Vidal}

Investigador Postdoctoral del Departamento de Sociología, Facultad de Ciencias Sociales, Universidad de Chile, Santiago, Chile jvergaravidal@uchile.cl | http://orcid.org/0000-0002-7712-4090

Diego Asenjo-Muñoz

Coordinador de Proyectos, Corporación Municipal de Renca, Santiago, Chile solaerama@gmail.com

Artículo recibido el 14 de enero de 2019 y aceptado el 07 de junio de 2019 DOl: https://doi.org/10.22320/07196466.2019.37.055.06 


\section{Resumen}

La proliferación de edificios en altura en las ciudades intermedias $y$ metropolitanas de Chile ha transformado tanto la fisonomía física y como la densidad simbólica de éstas. En este último plano, nuevas problemáticas han cambiado la configuración de los debates urbanos, de aquellos que se dan en espacios expertos, como de aquellos que se desarrollan fuera de ellos.

El presente texto investiga, en los debates y noticias registrados en la prensa chilena, la transformación contingente de los edificios de altura, desde objetos propiamente arquitectónicos a objetos heterogéneos, que se muestran abiertos a otros sentidos de asociación y, por tanto, a otras formas de politica y gestión.

Para ello se analizaron 482 piezas de prensa que mencionan a edificios en altura, publicadas por dos diarios de circulación nacional entre enero de 2009 y junio de 2017, con el fin de verificar cómo las temáticas que presentaban su localización urbana y la temporalidad de los hechos narrados revelaban la transformación de los marcos de sentido -más allá de lo arquitectónico- a los que son asociados dichos edificios.

\section{Resumo}

A proliferação de edifícios em altura em cidades médias e metropolitanas do Chile tem transformado tanto a fisionomia física como a densidade simbólica das mesmas. Em este último plano, novas problemáticas vêm alterando a configuração dos debates urbanos, tanto debates que acontecem em ambientes especializados, como debates que se desenvolvem fora de esses ambientes.

0 presente texto investiga, nos debates e notícias registrados pela imprensa chilena, a transformação contingente dos edifícios em altura, desde temas propriamente arquitetônicos a temas heterogêneos, que se mostram abertos a outros sentidos de associação e, por tanto, a outras formas de política e gestão.

Para o trabalho se analisaram 482 pesas de imprensa que mencionam edifícios em altura, publicados por dois diários de circulação nacional entre janeiro de 2009 e junho de 2017, com o fim de verificar como as temáticas apresentadas, sua localização urbana e a temporalidade dos feito narrados, dão conta da transformação dos marcos de sentido, diferentes aos arquitetônicos, e aos que são associados ao edifício.

\section{Abstract}

The proliferation of high-rise buildings has transformed the physical physiognomy and symbolic density of intermediate and metropolitan Chilean cities. At this level, new problems have changed the configuration of urban debates, of those that occur inside as well as outside of expert spaces, that is, academia and its associated communities of practice. The present article investigates the possible transformation of high-rise buildings in the debates and news recorded in the Chilean press: from strictly speaking architectural objects to heterogeneous objects that are open to other kinds of association and, therefore, to other forms of policy and management. To this end, 482 pieces of press mentioning high-rise buildings, published by two national daily newspapers between January 2009 and June 2017 were studied. The analysis examined how the themes presented, their urban location, and the temporality of the facts related gave an account of the transformation of the frameworks of meaning the buildings are associated with, in contrast to their architecture.
Palabras claves

Edificios en altura, periódicos, Chile, rascacielos; prensa arquitectónica
Palavras chaves

Edifícios em altura, periódicos, Chile, arranhacéus, imprensa arquitetônica

\section{Keywords}

High-rise buildings, newspapers, Chile, skyscrapers, architectural press 
FONDECYT 3170016, financiado por el Comisión Nacional de Investigación Científica y Tecnológica (CONICYT).

Véase: https://dle.rae. es/?id=IGVONxB

\section{Introducción*}

Los textos de arquitectura no han tenido solo un nombre para referirse a la tipología de los edificios en altura, les han llamado edificios altos (tall buildings), edificios de gran altura (high rise buildings), torres (towers), colectivos en altura, rascacielos (skyscrapers), entre otros (Sullivan, 1896; Glaeser, 2011; Steadman, 2014; Bernard, 2014). Tal indeterminación dentro de un conocimiento experto para nominar una tipología que le es propia puede llamar la atención, pero no es extraño que se produzca frente a un objeto complejo, continuamente debatido, interpretado y reproducido como éste.

Por su parte, la proliferación de edificios en altura o la verticalización de las ciudades chilenas, ha puesto en el foco de atención los desempeños poblacionales (Vergara, 2017) y económicos de esta tipología (Aguirre y León, 2007 ; 2008 ; Aguirre, Sandoval y Alliende, 2018), más que los desempeños propiamente arquitecturales (programáticos) de la misma.

Este artículo propone observar lo que se dice sobre los edificios fuera del campo de los conocimientos expertos, donde su indeterminación debiera ser mayor, pues los espacios para debatirlos y llegar a acuerdo sobre ellos no son explícitos ni formales, y no debiera acotarse solo a la forma de su nominación. La tarea es efectuada a través de lo que registra la prensa sobre los edificios en altura, y desde el entendido de que ésta se configura como un foro donde se reúnen diversos hechos y valores sobre aquellos.

Puede sonar forzado aceptar que un grupo de piezas de prensa sea considerado como un foro, dado que la imagen general que se tiene de uno está asociada a un espacio físico donde se da un debate, pero en realidad las acepciones de "foro" también incluyen la de "reunión de personas competentes en determinada materia, que debaten ciertos asuntos ante un auditorio que a veces interviene en la discusión", como sostiene el diccionario de la Real Academia Española', lo cual no limita ni la espacialidad ni la objetualidad de la reunión.

Los estudios de Ciencia, Tecnología y Sociedad (CTS) han utilizado la noción de foros (forums) para caracterizar los espacios donde se realizan las operaciones de definición de los objetos de conocimiento (Collins y Pinch, 1979; Callon y Rip, 1991; 1992; Callon, Lascoumes y Barthe, 2001). Les definen como espacios donde los actores llegan a acuerdo sobre las cosas y sus órdenes, y también como espacios donde es posible apreciar la movilización de los diferentes tipos de conocimientos que reconocemos como válidos, ya hayan sido configurados y adquiridos sistemáticamente, como es el caso de los conocimientos expertos, o por medio de la experiencia, como ocurre con la expertise.

Específicamente, los sociólogos Harry Collins y Trevor Pinch (1979) estudiaron los modos de denegación que operaban en la discusión científica para definir si un objeto, caracterizado como paranormal, era parte de una discusión seria o no. Para Collins y Pinch, estos modos de denegación operan de una manera implícita, en la cual las afirmaciones de conocimiento rivales sobre un objeto eran ignoradas por ortodoxia, de forma que ni siquiera eran impugnadas; o de una manera explícita, donde la necesidad de impugnación conllevaba la movilización de argumentos y actores en pos de definir, a favor o en contra, la controversia en torno al objeto de conocimiento. 
Ambos modos de denegación son prácticas que ocurren en espacios de debate o foros diferentes. La denegación implícita ocurre en foros constitutivos, donde imperan procedimientos formales que constituyen al objeto de conocimiento; por su parte, la denegación explícita se desarrolla en foros contingentes, donde se desarrollan acciones que no afectan la constitución del conocimiento objetivo y que pueden ser consideradas menos formales. Un foro contingente es también un foro heterogéneo, compositiva y temáticamente, donde el valor del conocimiento experto y de la experiencia está en debate.

Michel Callon y Arie Rip (1991; 1992) describieron como "híbridos" a foros con tales rasgos, puesto que configuraban situaciones altamente confusas en que los hechos y valores se relacionan de tal manera que ya no es posible distinguir entre la producción y diseminación de información o conocimiento y el proceso de toma de decisiones en sí mismo (Callon, 1988, p. 260), de modo que la relevancia del experto decae frente a los procesos de experiencia de las múltiples partes interesadas e implicadas en las situaciones; procesos que al mismo tiempo se convierten en un problema de negociación con efectos concretos como, por ejemplo, la emisión de normas o leyes.

En un trabajo posterior, Callon, Lascoumes y Barthe proponen que los foros hibridos

son espacios abiertos donde los grupos pueden reunirse
para discutir opciones técnicas que involucren al
colectivo, [son] híbridos porque los grupos involucrados
y los voceros que reclaman su representación son
heterogéneos, incluidos expertos, políticos, técnicosylaicos
que se consideran involucrados. También son híbridos
porque las preguntas y los problemas que se abordan, se
abordan en diferentes niveles enuna variedad de dominios,
desde la ética hasta la económica e incluso la fisiología,
la física nuclear y el electromagnetismo (2001, p. 18)

Los foros híbridos no solo son altamente heterogéneos en los valores que contienen y en los actores que convocan, son también políticamente complejos pues relacionan el rol del conocimiento experto y la experiencia con las formas de ordenamiento y negociación de los actores dentro de un espacio político. No solo fijan la naturaleza del objeto a partir de la equivalencia de valores entre el experto y la experiencia, sino que además negocian la gestión del objeto a partir de esto.

Observar estos espacios permite acercarse a los momentos donde es discutida la relación entre las entidades materiales y la sociedad, donde lo material adquiere agencia y configuración política. En este sentido, cada foro de debate, independiente de su forma y de su heterogeneidad, es responsable de la definición relacional de los objetos que discute, lo cual se expresa tanto en el tipo de controversias que reconoce y aborda, como en la articulación de acuerdos y arreglos en torno a ellas, como son los estándares y las tipologías.

En este caso se explora un tipo particular de foros. Aquellos que se organizan dentro de los medios de prensa, que dan cuenta de debates contingentes sobre objetos de conocimiento y donde encuentran lugar las voces de conocimiento experto y las voces de la experiencia. Estos foros se configuran en torno a los debates 
sobre hechos y valores que motivan objetos que se han vuelto controversiales. Ya sea el cambio climático, la industria minera, la provisión energética o los edificios en altura, las controversias y discusiones que motivan quedan inscritas en las piezas de prensa que, por separado y en su conjunto, informan sobre aquello que requiere ser discutido y (re)enlazado epistemológicamente al colectivo social.

En concreto, aqui se observa, en un conjunto de piezas de prensa que narran situaciones y controversias asociadas a edificios en altura, la manera cómo éstas se articulan en debates contingentes que versan, directa o indirectamente, sobre los diferentes modos en que las edificaciones son definidas y entablan relaciones con la comunidad. Se advierte, asimismo, cómo es el foro que articulan, que corresponde al modo en que los edificios en altura y la verticalización se hacen públicos y parte del debate social.

El texto que sigue expone, en primer lugar, la estrategia metodológica empleada para recopilar y analizar las piezas de prensa y, en segundo lugar, describe sus agrupamientos según la localización de los hechos que narran, su ubicación temporal y sus temáticas. En el tercer acápite, se discute el papel que cumplen estas temáticas en relación a los órdenes semióticos presentes en el debate social sobre los edificios de altura en la actualidad nacional, para luego concluir, en el cuarto acápite, que las temáticas operan no sólo como formas de agrupamiento de las piezas, sino también como modos de asociación semiótica.

\section{Metodología}

La estrategia metodológica en que se apoya esta investigación consideró que las piezas de prensa (noticias, artículos, notas, columnas] no constituyen únicamente registros de hechos y valores elaborados en torno a diversos objetos de conocimiento: son registros de una trama particular de debates, contingentes, pues su resultado está siempre abierto a ser configurado por cualquier tipo de conocimiento y por cualquier tipo de actor. Son también híbridos, ya que su composición convoca a diversos tipos de conocimientos expertos y no expertos, movilizados por variados tipos de actores, institucionales e individuales, que participan en ellos.

Dado este contexto, se estimó que la observación y análisis de las piezas de prensa que hacian mención a edificios de altura, podian ofrecer elementos para comprender cómo eran discutidas y configuradas las relaciones de hechos y valores que tenían como efecto la identidad de estos objetos y su significado relacional.

En lo que respecta los hechos, se observaron dos variables factuales: espacio, o el lugar o ciudad donde ocurría lo relatado en la pieza de prensa; y tiempo, o el año en que fue emitida la pieza. $Y$ en cuanto a los valores, se categorizaron las temáticas generales y especificas a las que hacian mención las piezas y se consideraron como variable de estudio.

Para la selección de las piezas se trabajó con aquellas publicadas en dos medios escritos chilenos de cobertura nacional (El Mercurio/Emol y La Tercera), entre 2009 y 2017, que narran situaciones asociadas especificamente a edificios en altura. Estas 


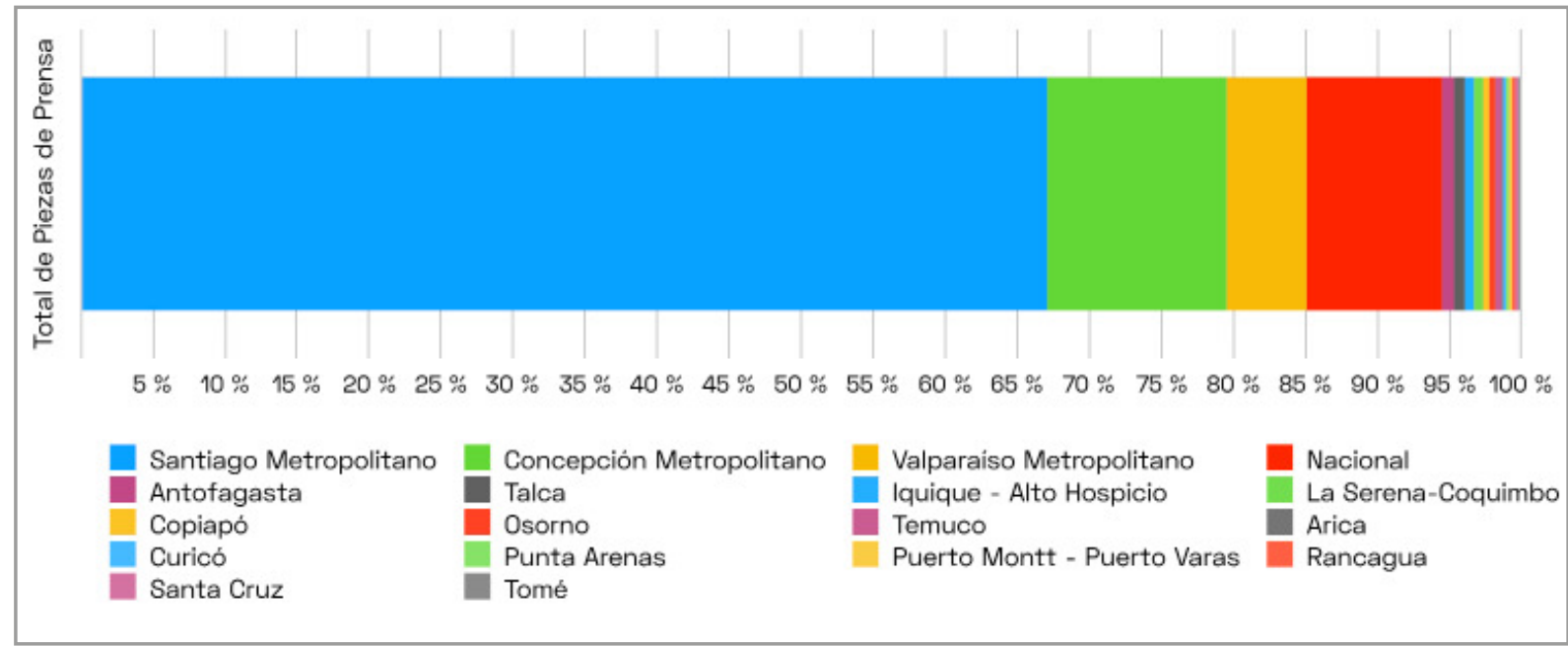

Figura 2. Porcentajes de Piezas de Prensa sobre edificios de altura según ciudad a la que hacen mención entre enero 2009 y junio 2017. Fuente: Elaboración de los autores, 2017.

piezas fueron escogidas utilizando el parámetro de búsqueda "edificio[s]" en los buscadores internos de los sitios web de estos medios, y se recopilaron solo las que hacian mención a casos nacionales.

Lo anterior limitó la búsqueda a la disponibilidad de archivos que, en el caso de La Tercera, solo llegan hasta 2009, e identificó 482 piezas publicadas en ambos medios, entre enero de 2009 y junio de 2017. A partir de esto, se elaboró una base de datos que fue analizada mediante estadísticos descriptivos presentes en el paquete SPSS 11. Su análisis permitió considerar los tipos de controversias identificadas en las piezas de prensa, los debates contingentes que configuran, su distribución geográfica y temporal. El siguiente apartado expone tales resultados.

\section{Los edificios en altura en la prensa chilena (2009-2017)}

De las 482 piezas de prensa examinadas, una vasta mayoría hace referencia a eventos sucedidos en edificios en altura de las tres ciudades metropolitanas del país, lo que evidencia el carácter fuertemente localizado de los hechos que dan pie a los debates y controversias respectivos. Asi, el 67,1\% menciona eventos ocurridos en Santiago Metropolitano; el 12,5\% a hechos ocurridos en el área del Concepción Metropolitano; y el 5,5\% a eventos ocurridos en comunas del Valparaíso Metropolitano (Figura 2). Esta distribución coincide con la importancia poblacional de estas tres zonas urbanas 


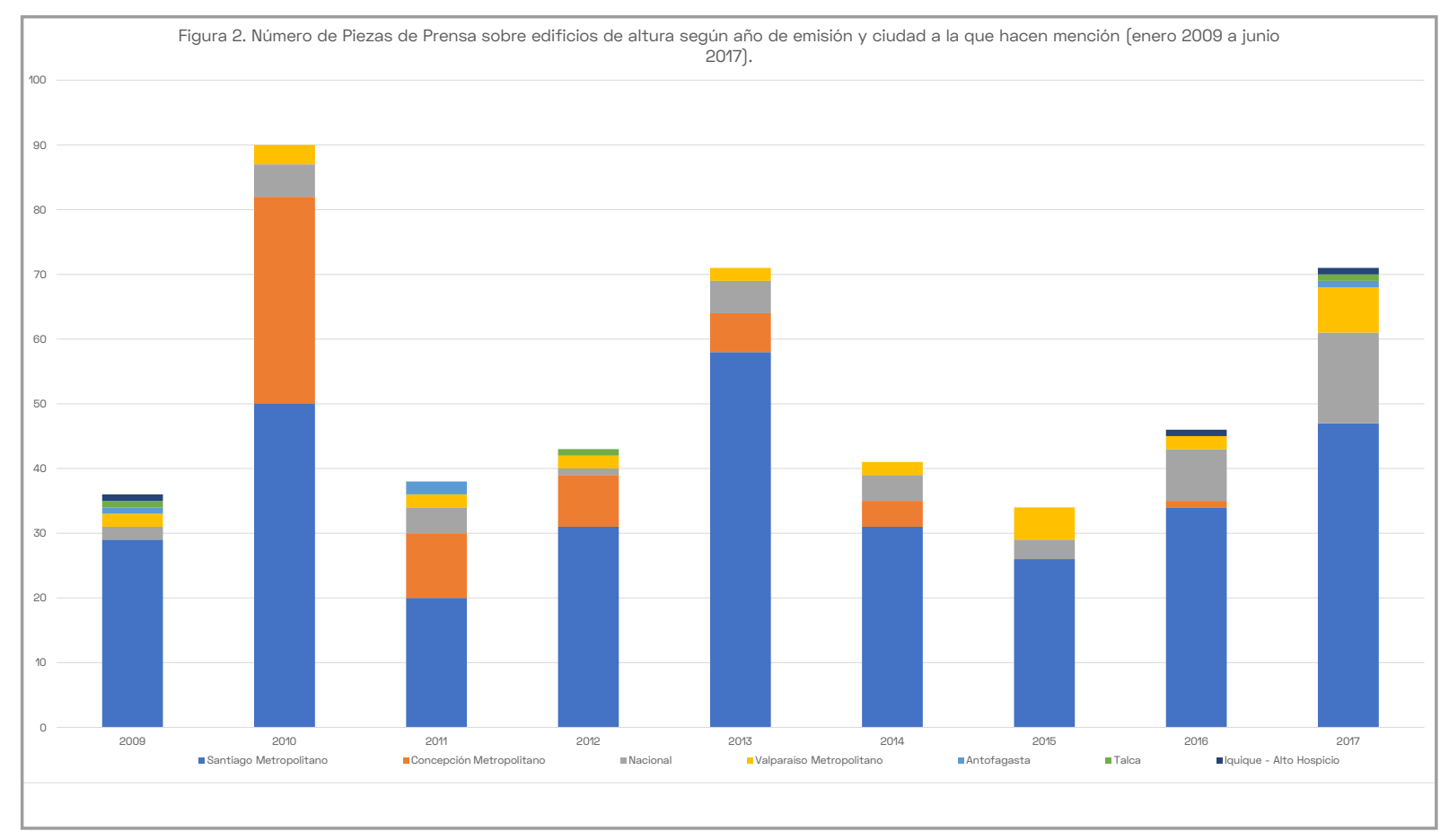

Figura 3. Porcentajes de Piezas de Prensa sobre edificios de altura según año de emisón y ciudad a la que hacen mención entre enero 2009 y junio 2017. Fuente: Elaboración de los autores, 2017.

dentro del país, lo que hace que sean más relevantes para los medios los eventos que ocurren allí. Por último, una pequeña fracción aborda eventos de escala nacional $(9,4 \%)$, que hacen mención a normas, regulaciones y fenómenos que afectan al conjunto de los edificios de altura del pais (Figura 2).

Desde el punto de vista cronológico, se aprecia que cerca de la mitad de las piezas estudiadas se concentran en tres años del periodo en cuestión, y en ellos se ubican tres eventos contingentes claves: el terremoto de 2010, las controversias judiciales del proceso de reconstrucción en 2013 y la controversia acerca de los guetos verticales en 2017 (Figura 3).

El terremoto de 2010, que afectó casi la totalidad de la zona centro sur del país, es claramente un evento notorio. Su pico se da en 2010, pero eventos relacionados directamente con él y edificios en altura continúan provocando noticias hasta el año 2014. Por lo mismo, la mayor cantidad de eventos y polémicas comunicadas a través de las piezas de prensa ocurren en los edificios de las ciudades que sufren con mayor intensidad los efectos sísmicos, la ciudad metropolitana de Concepción y la ciudad intermedia de Talca.

En estos lugares, las edificaciones en altura no solo sufrieron daños de diversa magnitud, algunas definitivamente se desplomaron, evidenciando errores en su cálculo estructural o en su construcción, o bien, la desobediencia de la norma antisísmica (o, incluso, todas las anteriores). Los debates al respecto se tornaron en seguida contingentes: comenzaron notificando los efectos del terremoto 


\begin{tabular}{|c|c|c|c|c|c|}
\hline Categoria & Temática & $\%$ & Categoria & Temática & $\%$ \\
\hline \multirow{11}{*}{$\begin{array}{c}\text { Accidentes } \\
\text { 38,1\% }\end{array}$} & Ascensor & 4,1 & \multirow{7}{*}{$\begin{array}{c}\text { Tecnologia } \\
\text { 11,9\% }\end{array}$} & Ascensor & 0,8 \\
\hline & Caída & 10,6 & & Bicicletas & 0,8 \\
\hline & Choque & 0,2 & & Construcción & 2 \\
\hline & Delito & 1 & & Energía & 2 \\
\hline & & & & Habitar & 5,7 \\
\hline & Derrube & 2 & & Protesta & 0,4 \\
\hline & Emergencia quimica & 2,9 & & Reciclaje & 0,2 \\
\hline & Explosión & 1 & \multirow{3}{*}{$\begin{array}{l}\text { Destino } \\
6,9 \%\end{array}$} & Edificio patrimonial & 1,8 \\
\hline & Gas & 1,4 & & Edificio público & 3,7 \\
\hline & Incendio & 14,9 & & Edificio privado & 1,4 \\
\hline & & & \multirow{4}{*}{$\begin{array}{l}\text { Ciudad } \\
6,1 \%\end{array}$} & Altura & 0,8 \\
\hline \multirow{5}{*}{$\begin{array}{c}\text { Terremoto } \\
18,4 \%\end{array}$} & Procesos judiciales & 7 & & Densidad & 4,1 \\
\hline & Reconstrución & 8,6 & & Permisos de edificación & 1 \\
\hline & & & & Vialidad & 0,2 \\
\hline & Robo & 1 & \multirow{5}{*}{$\begin{array}{l}\text { Delitos } \\
3,6 \%\end{array}$} & Sexual & 0,2 \\
\hline & Terremoto & 1,8 & & Bomba & 1 \\
\hline \multirow{3}{*}{$\begin{array}{c}\text { Mercado } \\
\text { inmobiliario } \\
\text { 14,5\% }\end{array}$} & \multirow{3}{*}{ Mercado inmobiliario } & \multirow{3}{*}{14,5} & & Crimen de sangre & 1,4 \\
\hline & & & & Robo & 0,4 \\
\hline & & & & Tomas & 0,6 \\
\hline
\end{tabular}

Tabla 1. Distribución de temáticas centrales en las piezas de prensa sobre edificios de altura observadas (enero 2009 a junio 2017). Fuente: Elaboración de los autores, 2017.

en las edificaciones, los eventos de limpieza y demoliciones, pero evolucionaron rápidamente al cálculo de los daños y las responsabilidades legales (Figura 3).

Las piezas de prensa emitidas en el año 2013 están relacionadas con esto último, pues dan cuenta del incremento de las inversiones inmobiliarias post terremoto, la construcción de nuevos y más altos edificios y los accidentes en estos procesos. La mayor cantidad de las piezas emitidas ese año $(81,7 \%)$ narran eventos localizados en Santiago Metropolitano, ciudad que apostó fuertemente por la edificación residencial en altura luego de que la gran mayoría de sus construcciones soportaran el embate de 8.9 grados de energía sísmica.

Como parte de esta materia es que emerge la controversia sobre los "guetos verticales", específicamente en cuanto al efecto densificador de los edificios residenciales de altura en la comuna de Estación Central y al rol de la planificación de los usos de suelo en ese sentido; lo cual correspondió a un alto número de piezas emitidas en 2017. Junto a ello, el 18\% de las piezas se refería a los problemas de regulación urbana y a las normas jurídicas involucradas, alcanzado así la mayor participación en todos los años observados y poniendo en evidencia el desplazamiento de los debates a la escala nacional que produjo la controversia antes señalada.

Al considerar la naturaleza de los eventos narrados por las piezas de prensa observadas fue posible articular siete categorías temáticas que permitieron clasificarlos y agruparlos: "Accidentes", 
"Ciudad", "Delitos", "Mercado inmobiliario", "Tecnología", "Destino del edificio" y "Terremoto". Esta última se ve, asociada a los eventos relacionados con el sismo de febrero de 2010. Dentro de este conjunto de categorías, se logró distinguir otras treinta y tres subcategorías temáticas que especifican aún más el tipo de evento narrado en la pieza de prensa (Tabla 1).

Las piezas de prensa que tratan sobre "Accidentes" relacionados con edificios de altura son las que acumulan una mayor representación porcentual, 38,1\%, y refieren a caídas o lanzamientos forzados desde los edificios, a incendios y a accidentes en ascensores, entre otros hechos. Le siguen las piezas que contienen noticias relacionadas con edificios de altura en el evento del "Terremoto" de 2010 , con un $18,4 \%$ del total, las cuales se abocan a los procesos de diagnóstico, reconstrucción y reparación de edificios afectados por el sismo, y a los procesos judiciales contra constructoras e inmobiliarias que tuvieron ocurrencia con posterioridad al terremoto.

La tercera categoría con mayor participación porcentual la ocupan piezas que narran situaciones que relacionan el "Mercado inmobiliario" con los edificios de altura (14,5\%). Estas tratan sobre la venta y compra de edificaciones, oficinas y viviendas, e informan las localizaciones de mayor rentabilidad. Por su parte, las piezas que versan sobre la relación de la "Tecnología" en los edificios en altura capturan el 11,9\% de las piezas, y se enfocan en normativas y controversias vinculadas con la naturaleza (inundaciones en bordes de río y mar], avances técnicos en la construcción y provisión de energía domiciliaria.

Las categorias con menor participación son aquellas que tocan el destino público, privado o patrimonial de los edificios de altura (6,9\%); la categoria "Ciudad" (6,1\%), que convoca mayoritariamente la participación de expertos; $y$, por último, la categoría de "Delitos" (3,6\%).

Considerando la agrupación según categorias antes expuesta, se verificó que, si bien las piezas de prensa muestran una predominancia estable, cada año señala acentos diferentes. Así, en el año 2010 las piezas de prensa que narraban situaciones sobre el terremoto capturaron casi el 50\% de las menciones a edificios de altura de ese año, lo cual desciende en los años siguientes, pasando otras temáticas a explicar las situaciones contingentes. De manera similar, las noticias relacionadas con el "Mercado inmobiliario" crecen notablemente en el año 2013 y 2017, años en que la discusión giró sobre los efectos posibles y reales del aumento del IVA en la compra de departamentos.

Punto aparte merecen las piezas de prensa asociadas a las temáticas de "Ciudad", que han incrementado su número en el año 2017, más que en todo el periodo observado y que alcanzan el 17\% de las piezas de este año, siendo superadas solo por las piezas de prensa ligadas al "Mercado inmobiliario". Esto tiene relación con la discusión sobre la deseabilidad de las edificaciones residenciales de altura, la densidad poblacional de los edificios (guetos verticales) y las consecuentes acciones de algunos gobiernos locales de limitar la altura de las edificaciones y revocar permisos de edificación.

Cada una de las temáticas recién expuestas agrupa los relatos sobre las contingencias que afectan a los edificios en altura en colectivos semióticos, dentro de los cuales los edificios 
Figura 3. Evolución de las temáticas centrales de Piezas de Prensa sobre edificios de altura observadas entre enero 2009 a junio 2017.

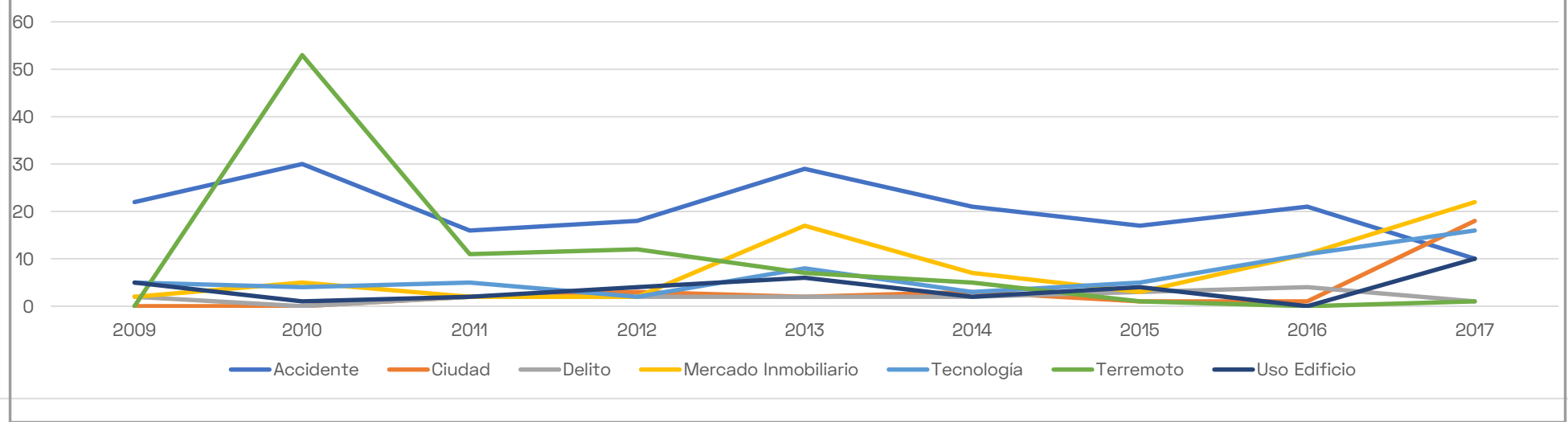

Figura 4. Evolución de las temáticas centrales de Piezas de Prensa sobre edificios de altura observadas entre enero 2009 a junio 2017. Fuente: Elaboración de los autores, 2017.

comparten tanto un significado como un valor similar; como ocurre cuando son escenarios de sucesos policiales o cuando representan suelo densificado dentro de la discusión urbana. La conformación de estos colectivos es lo que permite que sean reconocidos como una población particular e incluidos como tales en regímenes de gobierno. Pero hay que sumar a ello el carácter contingente que ostentan, dado que lo que las piezas de prensa narran constituyen situaciones en que los edificios están involucrados en algún tipo de controversia que enuncia un conflicto normativo, por lo que el o los regímenes de gobierno puestos en juego están siendo ajustados o evaluados en la situación observada.

El análisis de las piezas de prensa permite dar cuenta de la distribución geográfica de los edificios de altura, de la intensidad de las contingencias en que son incluidos y de las temáticas que los organizan. A partir de esto, es posible notar que los edificios en altura son debatidos constantemente bajo dos parámetros generales: su identidad objetual y su localización, las que parecen ser las bases de su carácter contencioso. Se les discute menos como objeto de arquitectura que como objeto económico; más sus efectos en la densidad o en el paisaje que los efectos de sus programas. A pesar de ser objetos técnicamente complejos, las situaciones y controversias en que se ven involucrados resultan ser más cotidianas, más administrativas y menos arquitecturales; más ligadas a la experiencia con y en ellos, que al conocimiento experto que los proyecta. 


\section{Foros heterogéneos, híbridos, contingentes}

Como se señaló anteriormente, las piezas de prensa constituyen registros de situaciones cuyos procedimientos para realizar afirmaciones sobre hechos y valores asociados a los edificios son menos rígidos que los de otros tipos de registros, como aquellos más propios del conocimiento experto. Por tanto, conforman una trama que coincide con lo que Collins y Pinch (1979) denominaron foros contingentes, aquellos donde los objetos discutidos son menos determinados por un solo tipo de variable y están más abiertos a ser configurados por más de un tipo de conocimiento.

De las variables observadas en las piezas de prensa, aquella que desagrega las categorías y las temáticas consideradas en éstas es la que resulta más interesante para entender la forma como se estructura el carácter contingente de este foro, en particular por su capacidad de mostrar la heterogeneidad del mismo. La diversidad de temáticas y categorías no es algo inherente a los edificios en altura, sino a la multiplicidad de sentidos a los que son simultáneamente asociados.

La heterogeneidad que evidencia no es otra que la propia de los órdenes semióticos, donde el valor asignado a un elemento depende de la diferencia con el valor asignado a otro. Si se asume que temáticas y categorías son en realidad valores, se ve claramente que el problema de la contingencia es que diversos valores son asignados, al mismo tiempo, a un mismo objeto.

El efecto de este fenómeno es claro cuando parece que hay más certeza en decir que los edificios de altura son un elemento propio de las grandes ciudades o un aspecto de la metropolitización de estas, que en afirmar que los edificios en altura son objetos solo concernientes a la economía, al urbanismo, al desarrollo inmobiliario, al derecho o a la arquitectura. En la primera afirmación no cabe duda porque no hay contingencia en ella, en la segunda sí, ya que ninguna opción parece incorrecta.

En un foro contingente, la heterogeneidad de los órdenes semióticos presentes se vuelve un indicador de la variación epistemológica del objeto (Law y Mol, 2002) y un problema para los acuerdos sobre su gestión. La preeminencia de algunas categorias y temáticas sobre otras, sin embargo, también indica sobre cuál de ellas sería más urgente ponerse de acuerdo, develando los aspectos que parecen más hostiles en la relación con los objetos y/o en los que estos nos hacen más vulnerables (Hommels, Mesman y Bijker, 2014).

Es esto lo que está implicado en la diferencia porcentual entre piezas de prensa de las categorias "Accidentes" (38,1\%) y la de "Ciudad" (6,1\%). Para el foro estudiado es más preocupante la seguridad en los edificios que su densidad o que la ocurrencia de delitos en ellos $(3,6 \%)$. Así, es posible asumir que habrá más acciones destinadas a lo primero que a lo segundo o a lo tercero, sin que ello implique que no van a haber acciones destinadas a ordenar la densidad de los edificios o la ocurrencia de delitos en ellos.

Cabe advertir también que es más difícil coordinar acciones masivas sobre la seguridad en edificios (no correr en escaleras, por ejemplo), que emitir una ordenanza que evite la densificación de suelos. Se discute más aquello sobre lo que es más difícil de ponerse de acuerdo, que aquello que resulta más contingente, lo cual es una forma de ordenar la heterogeneidad o las diferencias de las cosas (Law, 2009). 
Por último, es importante notar que, dentro de esta configuración contingente, las piezas de prensa que vehiculizan al conocimiento experto arquitectónico tienen una representación porcentual muy menor. En el foro estudiado, los edificios en altura casi no son discutidos de una manera arquitectónica. Basta indicar que se problematiza el aumento de su población, pero no la racionalidad o tipológica de las inmobiliarias. Ello colabora, desde este horizonte, a que los edificios en altura sean vinculados a otras explicaciones, como la seguridad, el rendimiento económico o la densificación urbana. Esa constante hibridez en la explicación de los objetos contribuye, por cierto, a que la condición contingente persista y a que no quede claro quién debe tomar decisiones respecto de ellos o sobre sus efectos: si los gobiernos, las inmobiliarias, el mercado, la arquitectura o todas las anteriores.

\section{Conclusión. El efecto contingente}

En la medida en que los edificios en altura proliferan en las ciudades chilenas y se denota su colaboración con la vulnerabilidad de estas, han ingresado estos en las discusiones contingentes de nuestra sociedad. Es, precisamente, lo que se ha observado al analizar las piezas de prensa que los mencionan, durante el periodo 2009-2017, desde un enfoque de foros de debate propio de los estudios de Ciencia, Tecnologia y Sociedad (CTS).

Consideradas en su condición de foro de debate, las piezas de prensa que se refieren a edificios en altura no abordan controversias de diseño: ni en relación con los programas de las viviendas, con los patrones de género o con la segregación que estos podrían provocar, ni sobre los diferenciales de apropiación de luz, calor, sombra y aire que, sin duda, la distribución de sus unidades en el espacio urbano produce. Por el contrario, se abocan a accidentes que ocurren en ellos, efectos que los sismos les producen, especulaciones que los involucran, decisiones sobre sus cambios de destino, crimenes que les tienen por escenarios, entre otros eventos que los involucran generalmente desde su exterior.

Lo que narran las piezas confirma que los objetos complejos, como los edificios en altura, están permanentemente colmados y rodeados de tensiones, variaciones, elementos no coherentes y prácticas heterogéneas, de modo que su estabilidad es sólo aparente (Harvey y Knox, 2012). A su vez, lo que las piezas de prensa evidencian es que tales controversias no son inherentes a los objetos mismos sino a la heterogeneidad de sentidos a los que son simultáneamente asociados, lo cual torna contingentes a los foros en los que son discutidos y a los mismos objetos.

Lo anterior implica que el efecto de la contingencia sobre los edificios en altura no afecta sus aspectos inherentes, pero si sus aspectos relacionales, específicamente, las asociaciones epistemológicas en las que son involucrados. Los edificios son sumados a discusiones sobre la criminalidad, el mercado, la geología, la población, entre otras, que no pueden resolver o en las que no pueden participar desde su diseño o desde sus repertorios políticos materiales (arquitecturales).

Esto sugiere un desplazamiento hacia un sentido relacional 
diferente, que tiene un énfasis en lo que los edificios en altura ayudan a ocurrir, sin ser algo inherente a ellos o, por lo menos, sin ser algo vinculable a la arquitectura. Se sugiere que dado lo anterior, tal sentido relacional podría ser infraestructural, toda vez que lo que hace es organizar la identidad de estos objetos mediante sentidos relacionados con las prácticas que se ve ocurrir en ellos (Star y Ruhleder, 1996).

Los modos en que los edificios en altura son hechos de arquitectura pueden estar radicados en su forma, su volumen, su materialidad, sus programas, su disposición en el trazado urbano, su habilitación tecnológica, entre otros aspectos socio-materiales a través de los cuales los edificios interaccionan como actores (no humanos) en situaciones relacionales directas y singulares. Sin embargo, los modos en que los edificios se tornan infraestructurales parecen no radicar en sus repertorios inherentes, sino en las asociaciones epistemológicas, económicas, normativas, materiales o urbanas en las que son contingentemente involucrados.

Se estima aquí que las piezas de prensa observadas configuran un foro en torno a hechos y valores que son ordenados de una manera aparentemente heterogénea, pero al volverlos contingentes y alejarlos de la determinación de un solo conocimiento experto (la arquitectura), no solo se revelan como epistemológicamente hibridos, abriéndose a ser explicados por otros conocimientos expertos y por otras experticias, sino que también quedan expuestos a ser vinculados a otros conjuntos relacionales. Del mismo modo como ocurre con las infraestructuras, que emergen como otro orden semiótico cuando la arquitectura desaparece de los debates.

Tal es quizás el efecto más claro de la condición contingente apreciada en las piezas de prensa sobre edificios en altura. Funciona como un indicador de que los objetos de los que hablan las piezas se encuentran abiertos a otros sentidos de asociación $y$, por consiguiente, a otras formas de políticas. Es interesante pensar lo que se abre en nuestras ciudades a partir de los efectos políticos de la proliferación de edificios en altura, pero también cabe considerar que mientras sigan siendo contingentes seguirán siendo parte de sus vulnerabilidades. 


\section{Referencias bibliográficas}

Aguirre C. y León, D. (2007). Análisis descriptivo de la evolución urbana de la Comuna de Ñuñoa, 2001 a 2006. Urbano, 10 (16), 60-72. Recuperado de http://revistas.ubiobio.cl/index. php/RU/article/view/377

Aguirre, C. y León, D. (2008). Aspectos causales del precio de departamentos nuevos en la Comuna de Ñuñoa, Santiago de Chile. Urbano, 11 (18), 59-67. Recuperado de http://revistas. ubiobio.cl/index.php/RU/article/view/348

Aguirre C., Sandoval, C. y Alliende, J. (2018). ¿Impacta la futura línea de metro en los precios de departamentos? Un estudio para Ñuñoa y Santiago Chile. Urbano, 21 (38), 84-95. DOl: https:// doi.org/10.22320/07183607.2018.21.38.07

Bernard, A. (2014). Lifted: A Cultural History of the Elevator. New York: New York University.

Callon, M. 1998. An essay on framing and over owing: economic externalities revisited by sociology. The Sociological Review: The Laws of the Markets 4 (1), 244-269. Oxford: Blackwell Publishers. DOl: https://doi.org/10.1111/j.1467-954X.1998.tb03477.x

Callon, M., Lascoumes, P. y Barthe, Y. (2001). Acting in an Uncertain World. An Essay on Technical Democracy. Cambridge: MIT Press.

Callon, M. y Rip, A. (1991). Forums hybrides et négociations des normes sociotechniques dans le domaine de l'environnement. La fin des experts et l'irrésistible ascension de l'expertise. Environnement, Science et Politique. Cahiers du Germes, 13, 227-238.

Callon, M. y Rip, A. (1992). Humains, Non- Humains: Morale d'une Coexistence. En J. Theys y B. Kalaoram, La Terre Outrage'e. Les Experts sont Formel!, pp. 140-156. París: Autrement.

Collins, H. M. y Pinch, T. J. (1979). The construction of the paranormal: nothing unscientific is happening. The Sociological Review, 27, 237-270. DOI:10.1111/j.1467-954X.1979.tb00064.x

Glaeser, E. (2011). El triunfo de las ciudades. Cómo nuestra mejor creación nos hace más ricos, más inteligentes, más ecológicos, más sanos y más felices. Madrid: Taurus.

Harvey, P. y Knox, H. (2012) The Enchantments of Infrastructure. Mobilities, 7 (4), 521-536. DOI: 10.1080/17450101.2012.718935

Hommels, A., Mesman, J. y Bijker, W. (Eds.). (2014). Vulnerability in Technological Cultures. New Directions in Research and Governance. Cambridge: MIT Press.

Law, J. (2002). Aircraft Stories. Decentering the Object in Technoscience. Durham: Duke University Press.

Law, J. y Mol, A. (2009). El actor-actuado: La oveja de la Cumbria en 2001. Política y Sociedad 45(3), 75-92. Recuperado de http:// revistas.ucm.es/index.php/POSO/article/view/POSO0808330075A

Star, S.L. y Ruhleder, K. (1996). Steps Toward an Ecology of Infrastructure: Design and Access for Large Information Spaces. Information Systems Research, 7 (1), 111-134. DOI: HTTPS://DOI.ORG/10.1287/ISRE.7.1.111

Steadman, P. (2014). Building Types and Built Forms. Leicestershire: Matador. Sullivan, L.H. (1896). The tall office building artistically considered. Lippincotts Magazine 57, 403-409.

Vergara Vidal, J. (2017). Verticalización. La edificación en altura en la Región Metropolitana de Santiago (1990-2014). INVI, 32 (90), 9-49. DOl: http://dx.doi.org/10.4067/S0718-83582017000200009 